\title{
Spike Proteins May be Dangerous
}

\author{
Manfred Doepp* \\ Holistic Center Head, Abtwil 9030, Switzerland \\ *Corresponding author: Manfred Doepp, Holistic Center, 13 Haupt St, Abtwil 9030, Switzerland. \\ To Cite This Article: Manfred Doepp. Spike Proteins May be Dangerous. Am J Biomed Sci \& Res. 2021 - 14(3). AJBSR.MS.ID.001990. \\ DOI: 10.34297/AJBSR.2021.14.001990.
}

Received: 㭗September 23, 2021; Published: 盋 October 01, 2021

\section{Opinion}

The mRNA vaccinations meanwhile are widespread in all industrialised countries. Surprisingly, there are countries where the vaccination rate is low and yet the incidence of COVID-19 infections is also low (see, among others, Uttar Pradesh in India with approx. $3 \%$ vaccination rate in a population of $260,000,000$ ). In the countries of the so-called "1st world", on the other hand, the vaccination rate is 60 to $70 \%$, without this having significantly reduced the number of infections. In Israel, the third vaccination campaign is currently underway (a fourth is planned), with hardly any reduction in the number of infections. One can conclude that there might be a mistake within the logic of the vaccinations. The official version is that it is a matter of mutants such as the Delta version, against which the vaccinations could be insufficiently successful. A third wave is expected, and then a fourth in winter, and so on.

The question arises whether the continuation of the previous vaccinations can be promising. The decisive factor in answering the question should be the extent to which the principle of integrating mRNA into DNA with an almost infinite production of spike proteins is considered to be life-saving. Can the immune system provide enough antibodies and lymphocytes to neutralize the spike proteins? Or is there a danger that cytokine storms can occur, which then overwhelm the organism? Can the increase in spike proteins in the blood trigger side effects that can no longer be controlled, such as overreactions in the sense of multiple microembolisms or macroembolisms? Basically, it should be noted that the COVID-19 viruses are not as dangerous as was initially thought. They are viruses from the long-known groups of SARS viruses and Corona viruses, which mankind has long learned to deal with without there having been an epidemic or a pandemic. What is decisive and new is the appearance of the spike proteins. They are able to block the ACE-2 receptors that exist everywhere in the organism. And it is this process that makes COVID-19 viruses dangerous. They act as transporters for their spike proteins, so to speak, and bring them everywhere in the body where they can dock and carry out their dangerous, because blocking, work.

In the first few months, infections with COVID-19 viruses led to death rates (apart from patients over 80 years of age) of less than $0.1 \%$ of those affected. This is within the range of the annual influenza waves. Looking at the excess mortality rates in the last 18 months, they were higher than the long-term average only in very old patients. The death rates from other common diseases such as cancer and heart attacks even decreased to the extent that hospital beds were hardly available for these patients and - apparently not sufficiently indicated - operations had to be postponed. What can be the consequence of widespread mRNA vaccinations? As far as the immune system reacts normally, with neither undernor overreactions, the patients can live with it. They are neither spreaders nor even super-spreaders of spike proteins. The situation is different if an immune aberration exists.

The vaccinated can become spreaders who load their environment with spike proteins in any concentration. This can occur via exhaled air in the form of aerosols as well as via other body fluids. The most sensitive entry points are not the throat or the nasal mucosa, as these have an excellent defense system, but the eyes. In the area of the pupils and the conjunctiva, the body has only a minimal defense system at its disposal, and entry for spike proteins is possible without any problems. In this respect, it is counterproductive if the masks protect the mouth and nose while the eyes remain unprotected. Glasses like those worn by skiers would be indicated. 


\section{Conclusion}

mRNA vaccinations can be dangerous if, as a consequence of an unchecked production of spike proteins, the immune system does not react adequately, but over- or underreacts, which, on the one hand, can trigger a multitude of ACE-2 receptor blockades in vaccinated persons, and, on the other hand, can cause a hazard to their environment by transferring spike proteins. Under certain circumstances, such spreaders can become super-spreaders. 